\title{
Using the Ilizarov technique for the correction of coxa vara
}

\begin{abstract}
Background and objective: Coxa vara was treated previously by subtrochanteric and inter-trochanteric valgus osteotomies with internal fixation. Recently; more attention was paid for achieving corrections of limb deformities and length discrepancies through less invasive means using external fixator systems including llizarov fixation. This study aimed to describe the performance of llizarov fixation technique in the surgical correction of coxa vara of different etiologies and limb lengthening.

Methods: In this case series study, nine patients (11 hips) with coxa vara of different etiologies were treated surgically by sub-trochanteric osteotomy done percutaneously with the use of Ilizarov fixation device, in 15 months at Erbil teaching hospital. Patients between four and ten years of age with coxa vara with neck - shaft angle less than $120^{\circ}$ or Hilgenreiner epiphyseal angle of more than $40^{\circ}$ were included in this study.

Results: All osteotomies achieved the targeted correction in the Hilgenreiner -epiphyseal angle and the neck-shaft angle with a $P$ value $<0.001$. All osteotomies healed primarily and on follow-up, no intra-operative complications developed, and no patient needed the post-operative blood transfusion. Three patients needed limb lengthening, one patient needed shelf osteotomy, and two patients had superficial pin tract infection. Four patients had postoperative knee stiffness which resolved completely. Radiological analysis revealed a maintained improvement in the Hilgenreiner-epiphyseal angle and neck-shaft angle.

Conclusion: Ilizarov technique is a safe and less invasive technique for the surgical management of coxa vara of different etiologies and limb length discrepancy.
\end{abstract}

Keywords: Coxa vara; Ilizarov; Subtrochanteric osteotomy.

\section{Introduction}

Coxa vara, classically defined as a femoral neck-shaft angle of fewer than 120 degrees, is relatively uncommon, occurring in approximately one per 25000 children. This deformity results from a heterogeneous group of conditions that can be classified as congenital, developmental, dysplastic, and traumatic. The natural history of coxa vara may be debilitating as the child develops progressive limb length discrepancy, limping, pain, abductor weakness, and restricted motion. Secondary acetabular dysplasia and genu valgus may compound the situation. ${ }^{1-3}$ Historically, treatment has evolved from non-operative (which was found to be ineffective) to surgical, with both subtrochanteric and intertrochanteric valgus osteotomies with internal fixation. ${ }^{4,5}$ Despite well-performed osteotomies, recurrence is cited in the literature ranging from 30 to $70 \% .{ }^{6-10}$ Recently; more attention was paid for achieving corrections of limb deformities and length discrepancies through less invasive means using external fixator systems including Ilizarov fixation for the correction of proximal femoral deformities. ${ }^{1}$ This study describes the performance of Ilizarov fixation technique in the surgical correction of coxa vara of different etiologies and limb lengthening.

* Emergency Hospital, Directorate of Health, Erbil, I raq.

** Department of Surgery, College of Medicine, Hawler Medical University, Erbil, I raq. 


\section{Methods}

This case series study was done in 15 months between November 2014 and February 2016. Patients included in this study were presented to the orthopedic consultant clinic of Erbil teaching hospital within the period of the study. Their chief complaint was limping with minimal or no pain. Clinical examination was done to determine the range of movements of hip and knee joints, lower limb length discrepancy by measuring the distance from anterior superior iliac spine to the medial malleolus of the tibia using the tape measure, and to find out any stiffness of hip and knee, and any deformity of the back. Trendelenburg test was performed; x-ray of the affected hip was performed including $A P$ and lateral views. Suitable biochemical studies were done for diagnosing the cause of coxa vara and making a suitable plan for treatment. Patients between four and ten years of age with coxa vara with the neck-shaft angle (NS angle) less than $120^{\circ}$ or Hilgenreiner epiphyseal angle (HE angle) of more than $40^{\circ}$ were included in this study. Patients with general medical status unfit for general anesthesia, patients with hip or knee stiffness, those with chondrolysis of the hip joint with obliterated joint space, and active infection of the hip or femur, were excluded from the study. Nine patients were included, three boys and six girls, their average age was $7.33 \pm 2.33$ years with a range of 4-10 years. In two patients both limbs were involved; the right side was involved in four limbs. History was taken from the patients' parents including family history, drug history, and surgical history. Clinical examination, radiological investigation, and biochemical tests revealed that all patients had abductor lurch with Trendelenburg's test positive pre-operatively, limb discrepancy with an average of $3.3 \pm 1.8 \mathrm{~cm}$. All patients had $\mathrm{HE}$ angle of more than 40 degrees with an average of $62.7 \pm 11.5$ degrees, and NS angle defect of fewer than 120 degrees with an average of $104.8 \pm 8.7$ degrees (Table 1). The clinical preoperative examination also revealed that three patients (5 limbs) had a limblength discrepancy of more than $2 \mathrm{~cm}$. Three patients had coxa vara as a result of avascular necrosis of the head of the femur (resulted from a closed reduction of DDH with P.O.P. spica and adductor tenotomy in two patients while the other patient was with Perthe's disease treated by excessive varus osteotomy). Three patients had developmental coxa vara, one patient with nutritional rickets, and two patients with $\mathrm{DDH}$ and residual coxa vara after surgical correction, indicating that 6 of the patients had acquired coxa vara as shown in Table 2. The patient with nutritional rickets was operated on after normalization of the blood indicators (alkaline phosphatase, serum calcium, serum phosphate, and vitamin D). Four patients $(44.4 \%)$ had previous operations including the two with

Table 1: Patient's demographic and clinical data.

\begin{tabular}{lc}
\hline Variables & \\
\hline Age & $7.3 \pm 2.3$ \\
Average (Mean \pm SD) in years & $4-10$ \\
Range in years & \\
Gender & $0.5: 1$ \\
Male: Female ratio & $62.7 \pm 11.5$ \\
Pre-op. HE angle degrees (Mean \pm SD) & $104.8 \pm 8.7$ \\
Pre-op. NS angle degrees (Mean \pm SD) & \\
\hline
\end{tabular}


a history of open reduction of $\mathrm{DDH}$ who developed coxa vara after varus osteotomy, one patient with Perthe's disease had coxa vara as a sequel of varus osteotomy to achieve containment (lateral pillar type C), and two patients with bilateral DDH treated by adductor tenotomy and closed reduction under general anesthesia with POP spica; they developed avascular necrosis with coxa vara.

Operative Procedure:

Under general anesthesia, the patient was placed in supine position on a radiolucent orthopedic table with a folded sheet placed in the midline, under the sacrum. The lower extremity to be operated on was prepared, and free draped. The operative technique used is that described by Paley and Standard ${ }^{11}$ with slight modification. Based on the preoperative clinical and radiographic findings, the affected limb was held in a "hip neutral position as the deformity was varus. In cases with flexion and retroversion, the hip was held in adduction position with slight flexion and external rotation. In this position of the limb, a true AP of the involved hip was reproduced using $\mathrm{C}$-arm monitor; hip abduction was examined, and adductor tenotomy was performed for three patients who had the limitation of abduction less than $45^{\circ}$ with tight adductor muscles. When proximal femur derotation and valgus osteotomy are needed, proximal pins were inserted into the proximal femur with the hip in the position in which it will lie after the correction. For the correction of varus deformity and anteversion, the femur was adducted (crossing the other limb) and internally rotated thus placing the hip in a true neutral position. Two pins $(4 \times 150 \mathrm{~mm})$ were inserted from superolateral (nearby the greater trochanter), avoiding injury to the epiphysis to the inferiomedial direction (nearby the lesser trochanter); the pins are parallel to each other and parallel to an imaginary line from the tip of greater trochanter to the center of femoral head (proximal joint orientation line). The half ring was attached proximally and connected to the pins by bolt and nuts, then another two distal pins $(4 \times 150 \mathrm{~mm})$ was applied $5 \mathrm{~cm}$ distal to the lesser trochanter and connected by the second half ring. Those pins were perpendicular to the anatomical axis of the femur, as described in other literature..$^{9,11,12}$ A longitudinal incision of one $\mathrm{cm}$ length was made at the level of the proposed osteotomy site at the subtrochanteric area, between the two arches. Under fluoroscopic control, a $3.5 \mathrm{~mm}$ drill bit was used to create drill holes of the lateral

Table 2: Description of the clinical condition of the patients.

\begin{tabular}{llc}
\hline & Clinical findings & Number of patients \\
\hline 1) & Abduction lurch & 9 patients $(11 \mathrm{limbs})$ \\
2) & Trendelenburg's test positive & 9 patients $(11 \mathrm{limbs})$ \\
$3)$ Limb length discrepancy: & \\
$<2 \mathrm{~cm}$ & 6 patients $(6 \mathrm{limbs})$ \\
$>2 \mathrm{~cm}$ & 3 patients $(5 \mathrm{limbs})$ \\
Developmental coxa vara & 3 patients \\
Acquired coxa vara: & 6 patients \\
Avascular necrosis & 3 patients \\
Rickets & 1 patient \\
D.D.H. & 2 patients \\
\hline
\end{tabular}


https:/ / doi.org/ 10.15218/ zjms.2019.002

near) cortex. The drill bit was directed anteriorly and posteriorly to create multiple perforations of the medial (far) cortex. The transverse osteotomy was completed by connecting the drill holes with quarter-inch osteotomes. First, we started with the correction of retroversion (in developmental coxa vara) or anteversion (in patients with DDH or Perthe's disease) if needed. (Correction of retroversion was done in two

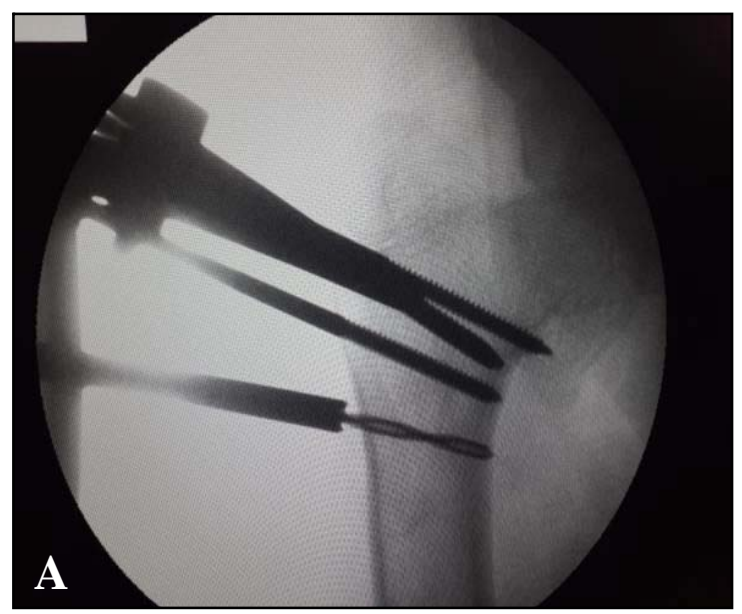

cases while correction of anteversion was done in three cases, followed by lateral translation of the distal segment. Finally, the correction of varus deformity was done by angulation of the proximal segment into a valgus position bringing the NS angle to 135 degrees, so the lateral cortex of the proximal segment was wedged in the medullary cavity of the distal segment (Figures 1, 2).

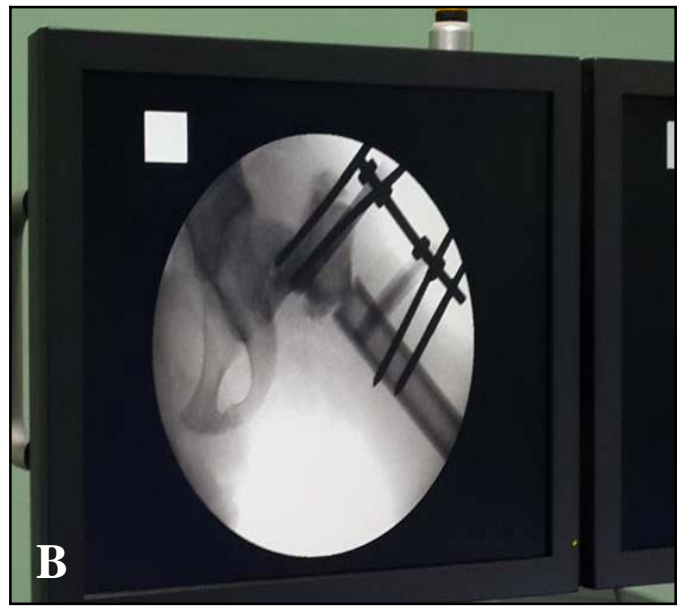

Figure 1 (A, B): Applying the proximal and distal Schanz pins under fluoroscopic guidance.
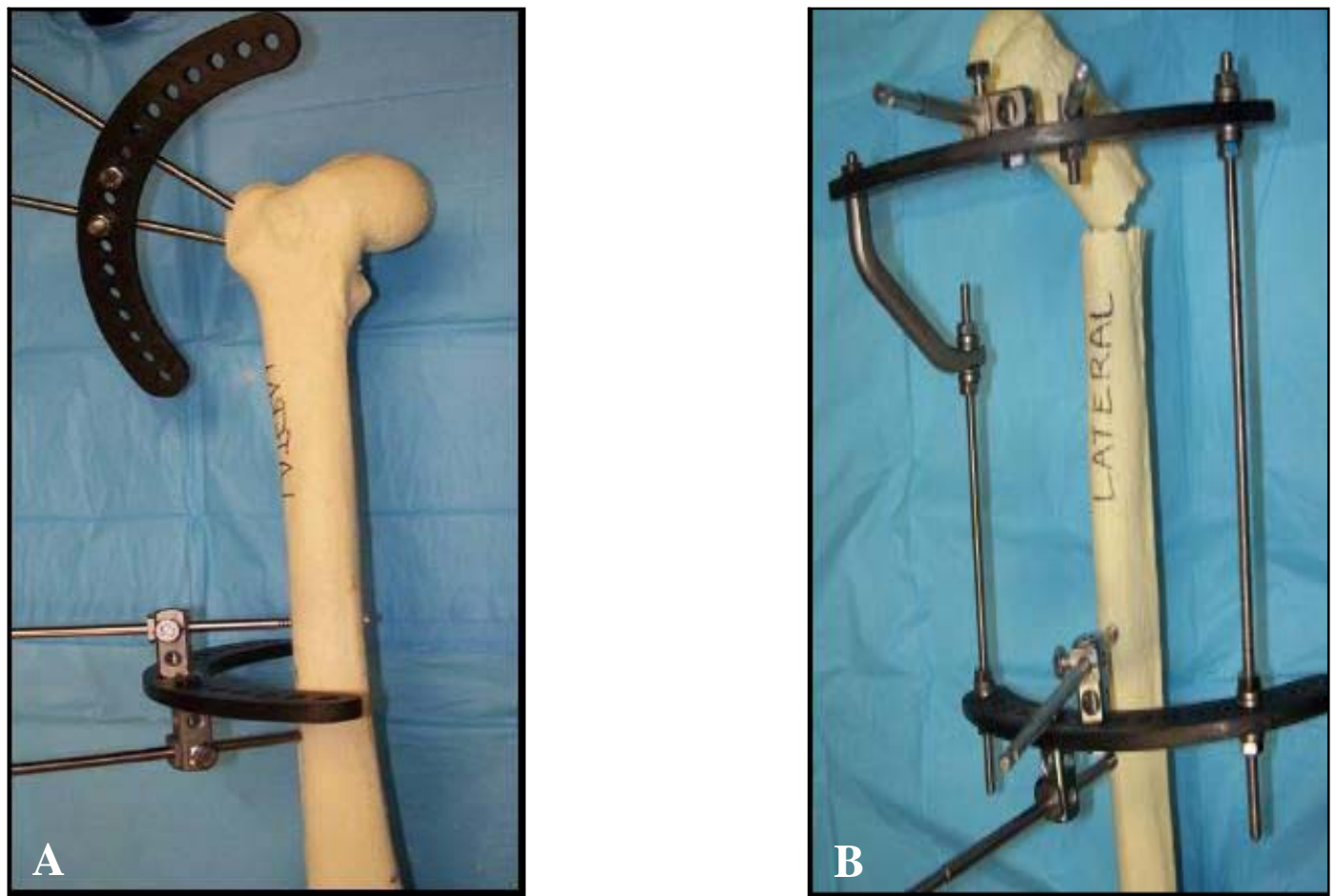

Figure 2 (A, B): Demonstration using sawbone showing the direction of pins and the site of osteotomy. 
https:/ / doi.org/ 10.15218/ zjms.2019.002

Three patients $(5$ limbs) with limb discrepancy was more than two $\mathrm{cm}$ distal lengthening was done using two rings distally above the level of femoral condyles. Each ring was attached to two wires and one pin, the rings were parallel to the knee joint line, and were parallel to each other at the end of the procedure and were connected together using a motor bar for

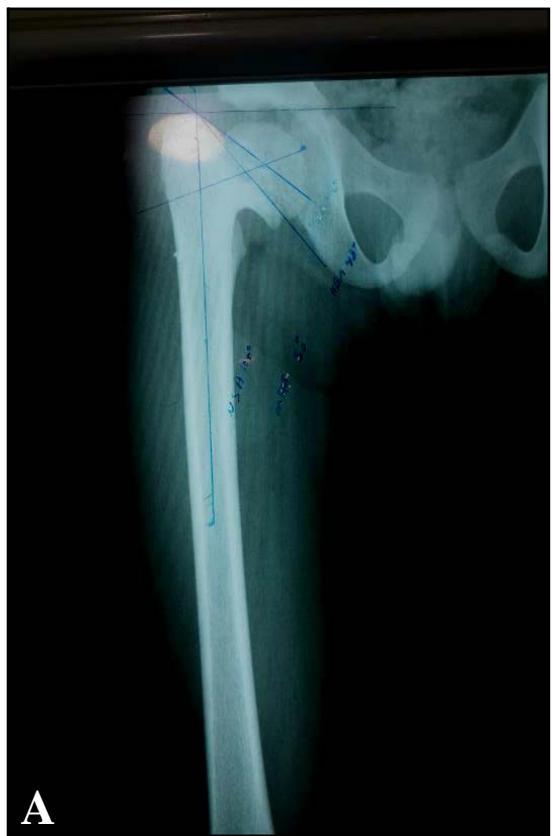

distraction and lengthening. An incision of one $\mathrm{cm}$ of the skin between the two distal rings was done followed by drilling circumferential holes in the supracondylar area by $3.8 \mathrm{~mm}$ drill then cutting the bone by a quarter inch osteotomy. The proximal frame was connected to the distal frame by three bars (Figures 3 and 4).

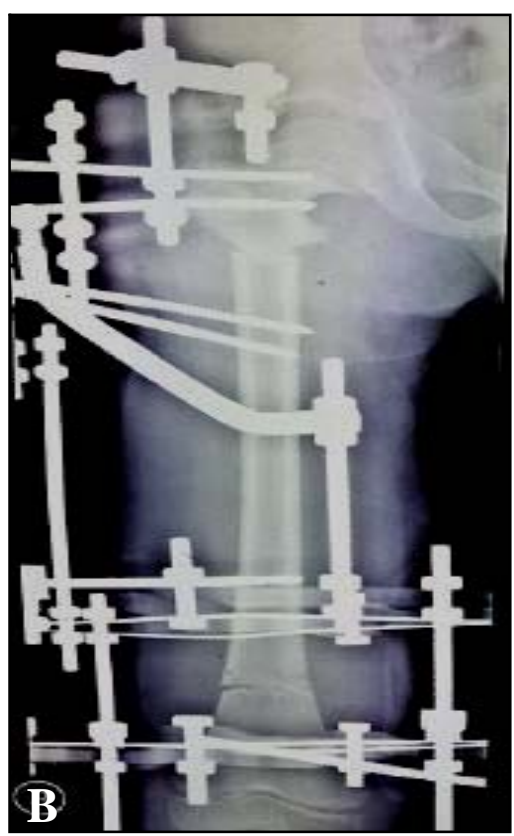

Figure 3 (A, B): Pre and postoperative imaging.

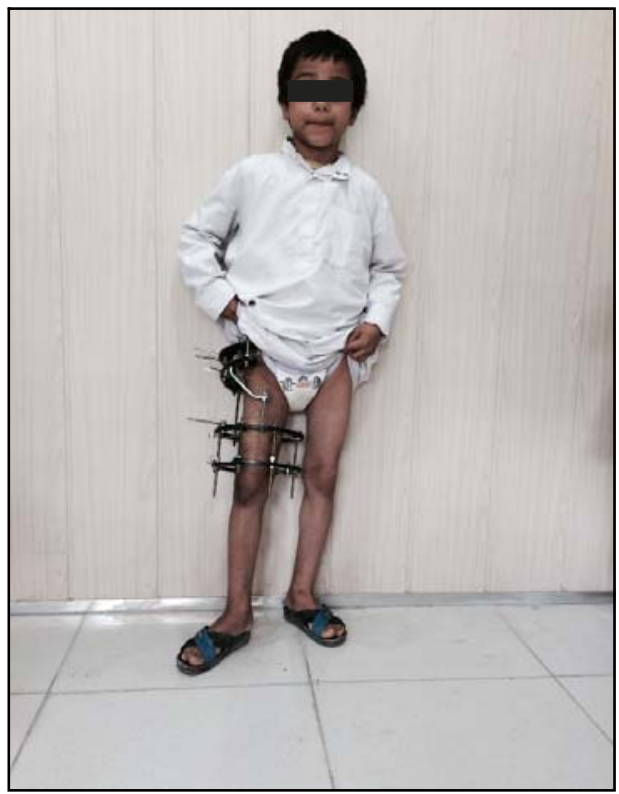

Figure 4: 9 years old child with Coxa vara and shortening up to $4 \mathrm{~cm}$ treated by the llizarov technique. 
Postoperatively, oral antibiotics were prescribed for ten days with analgesia. Physiotherapy started after 14 days including quadriceps exercise and flexion of the knee joint and walking with crutches. Follow-up was done including clinical examination and $x$-ray imaging (AP and lateral) of the hip that had been operated on immediately, then after one month, three months, six months, nine months, 12 months, and 15 months postoperatively. All patients had at least a year of follow-up. The llizarov fixation was removed after 3-5 months under general anesthesia after confirming radiological healing of the osteotomy site with no non-union or malunion. Physiotherapy was started immediately after the removal of the Ilizarov fixation. The study was approved by the research ethics committee of the
College of Medicine at Hawler Medical University. Written informed consent was obtained from the patient's parent before surgical treatment. Data were analyzed using the statistical package for the social sciences (version 19). Paired t-test was used to compare means before and after the operations. A $P$ value of $\leq 0.05$ was considered statistically significant.

\section{Results}

The average follow-up was $13.5 \pm 2.1$ months. No revision was needed for any patient. All the osteotomies achieved the targeted $\mathrm{HE}$ angle of fewer than 40 degrees with an average of $35.63 \pm 2.37$ (Figure 5), and the targeted NS angle of more than 130 degrees with an average of $136 \pm 2.56$ degrees (Figure 6).

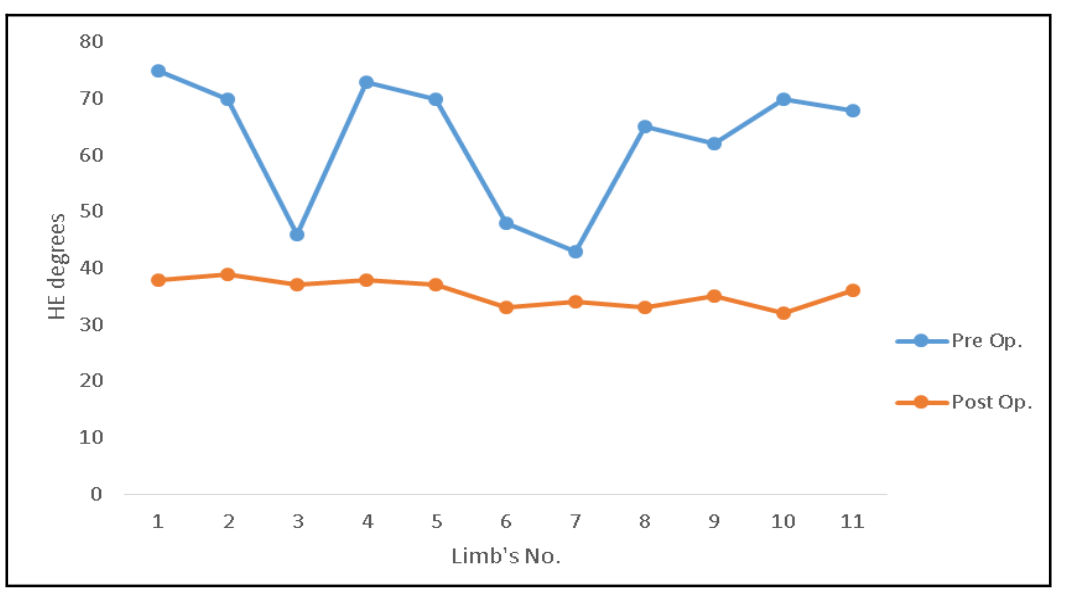

Figure 5: Comparison between the Hilgenreigner- epiphyseal angle (in degrees) in the 11 limbs and coxa vara. Pre and post-operative.

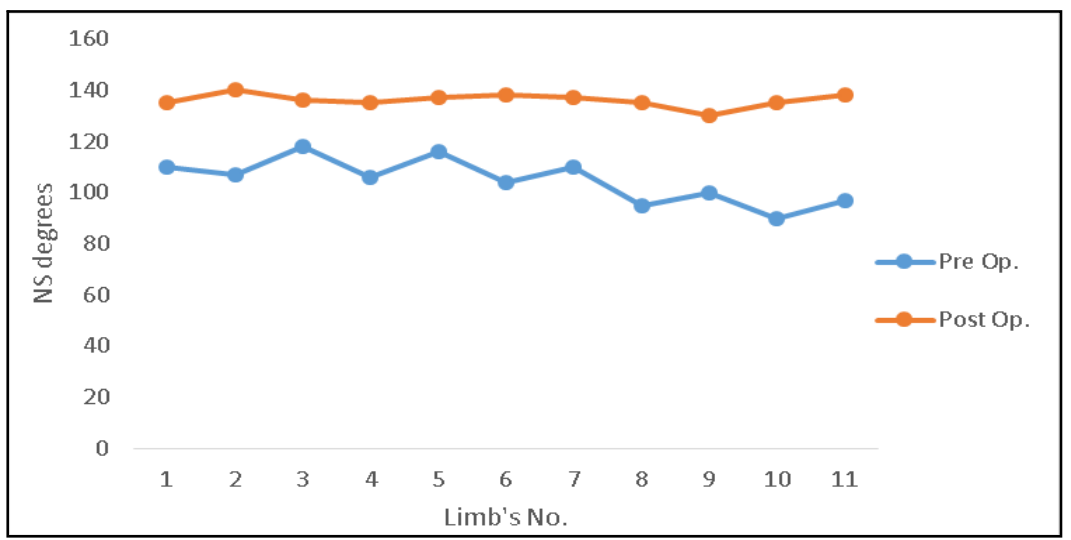

Figure 6: Comparison of the neck-shaft angle (in degrees) in the 11 limbs with coxa vara. Pre and post-operative. 
The average correction in the $\mathrm{HE}$ angle was $27.45 \pm 10.2$ degrees $(P<0.001)$, and that in the NS angle was $29.45 \pm 11.96$ degrees $(P<0.001)$. Details are shown in Table 3. No intra-operative complications including fractures or neurovascular injury happened. No patient needed a postoperative blood transfusion. No limb discrepancy was reported. Three patients $(33.3 \%)$ (5 limbs) needed lengthening which was done at the same operation. One patient $(11.1 \%)$ needed shelf osteotomy to achieve containment of the femoral head, and two patients (22.2\%) had superficial pin tract infection treated with oral antibiotics and local irrigation with normal saline impregnated with antibiotics; no intravenous antibiotics were needed, and no further sequelae happened. Four patients $(44.4 \%)$ had postoperative ipsilateral knee stiffness completely resolved with regaining of the full range of motion by physiotherapy within two months following removal of the llizarov fixation.

\section{Discussion}

In this study, no revision was needed for any patient, all the osteotomies achieved the targeted $\mathrm{HE}$ angle of fewer than 40 degrees with the average of $35.63 \pm 2.37$ degrees, and the targeted NS angle of more than 130 degrees with an average of $136.00 \pm 2.56$ degrees. These results agree with those of Sabharwal et al., ${ }^{12}$ who achieved an average postoperative $\mathrm{HE}$ angle of 33 degrees and an average postoperative NS angle of 137 degrees. In Hefney et al. $^{9}$ study, the average postoperative HE angel was 34 degrees, and the average postoperative NS angle was 133 degrees. In our study, three patients had developmental coxa vara, and six patients had acquired coxa vara including; Perthe's disease, avascular necrosis as a result of a close or open reduction of $\mathrm{DDH}$ and cricket. On the other hand, Sabharwal et al. ${ }^{12}$ study was done on five patients with developmental coxa vara only, while Hefney et al. $^{9}$ study was done on ten patients, seven had developmental coxa vara, two patients had fibrous dysplasia, and 1 had a proximal femural focal defect. However, both of the studies recommended using the llizarov technique to correct coxa vara in slipped capital epiphysis, Perthe's disease, and $\mathrm{DDH}$. Based on clinical examination all patients were within $1 \mathrm{~cm}$ of the contralateral limb length at latest follow-up; a finding which agrees with that of Sabharwal et al. ${ }^{12}$ However, three patients (5 limbs) with limb discrepancy of more than $2 \mathrm{~cm}$ were not expected to be leveled with correction of coxa vara only; therefore, they needed lengthening done at the same operation. Sabharwal et al. ${ }^{12}$ did not perform distal osteotomy for lengthening while Hefney et al. $^{9}$ performed distal osteotomy and lengthening on four patients. One patient $(11.1 \%)$ with Perthe's disease needed shelf osteotomy to achieve containment of the femoral head as a result of coxa magna. Two patients

Table 3: Correction achieved in HE and NS angles in the 11 limbs.

\begin{tabular}{lcc}
\hline Variables & HE angle (Mean \pm SD) & NS angle (Mean \pm SD) \\
\hline Pre-operative degrees & $62.7 \pm 11.5^{\circ}$ & $104.8 \pm 8.7^{\circ}$ \\
Post-operative degrees & $35.6 \pm 2.3^{\circ}$ & $136.0 \pm 2.5^{\circ}$ \\
Correction degrees & $27.4 \pm 10.2^{\circ}$ & $29.4 \pm 11.9^{\circ}$ \\
$P$ value & $<0.001$ & $<0.001$ \\
\hline
\end{tabular}


$(22.2 \%)$ had superficial pin site discharge treated by oral antibiotics with no further sequelae. Sabharwal et al. ${ }^{12}$ reported that superficial pin tract infection was common in their patients, but no patient needed intravenous antibiotics with no sequelae happened in any patient. Five (33.3\%) patients with superficial infections in Hefney et al. ${ }^{9}$ study were treated with an intravenous administration of antibiotics and frequent dressing changes and were healed uneventfully. Four patients (44.4\%) had postoperative ipsilateral knee stiffness completely resolved with regaining full range of motion by physiotherapy within two months following the removal of the Ilizarov fixation. This finding was similarly reported by Sabharwal et al. ${ }^{12}$ who reported that some restriction of knee motion was commonly seen while the patients had the external fixator, full knee mobility was achieved within four weeks of fixator removal in all patients. Other workers reported that the llizarov technique has potential advantages over commonly used open techniques and provides an available alternative to currently applied methods used for fixation of proximal femoral osteotomies. However, the technique has certain drawbacks including the chance for pin tract infection, the radiation exposure due to using fluoroscopic guidance and the need for experience with the use of Ilizarov apparatus. ${ }^{9,12}$

\section{Conclusion}

Ilizarov technique proved to be a safe alternative for surgical correction of both coxa vara and limb discrepancy at the same session with early mobilization, less amount of blood loss, short hospital stay, and no need for another major surgical exposure for removal of the internal fixation with lower potential for deep infection.

\section{Competing interests}

The authors declare that they have no competing interests.

\section{References}

1. Hefny $H$, Elmoatasem EM, Nassar W. Valgus osteotomy by external fixation for treatment for developmental coxa vara. Strategies Trauma Limb Reconstr 2013; 8(3):161-7.

2. Benson M, Fixsen J, Macnicol M, Parsch K. Children's orthopedics and fractures. $3^{\text {rd }}$ ed. London: Springer International Publishing; 2010.

3. Sabharwal S. Pediatric lower limb deformities. Principles and techniques of management. $1^{\text {st }}$ ed. London: Springer International Publishing; 2016.

4 EL-Sobky T. Subtrochanteric valgus osteotomy in developmental coxa vara. Indian J Orthop 2011; 45(4):320-3.

5. Beals RK. Coxavara in childhood: evaluation and management. J Am Acad Orthop Surg 1998; 2:93 -9 .

6. Günther CM, Komm M, Jansson V, Heimkes B. Midterm results after subtrochanteric end-to-side valgization osteotomy in severe infantile coxa vara. J Pediatr Orthop 2013; 33(4):353-60.

7. Burns KA, Stevens PM. Coxa vara: another option for fixation. J Pediatr Orthop B 2001; 10(4):30410.

8. Widmann RF, Hresko T, Kasser JR, Millis MB. Wagner multiple K-wire osteosynthesis to correct coxa vara in the young child: experience with a versatile 'tailor-made' high angle blade plate equivalent. J Pediatr Orthop B 2011; 10(1):4350.

9. Hefney HM, Elmoatasem EM, Nassar WA. Treatment of coxa vara utilizing an external fixator. Egypt Orthop J 2015; 50:68-72.

10. Carroll K, Coleman S, Stevens P. Coxa vara: surgical outcomes of valgus osteotomies. J Pediatr Orthop 1997; 17(2):220-4.

11. Paley D, Standard SC. Lengthening reconstruction surgery for congenital femoral deficiency. In: Rosbruch SR, llizarov S, editors. Limb lengthening and reconstruction surgery.New York: Informa Health Care; 2007.

12. Sabharwal S, Mittal R, Cox G. Percutaneous triplanar femoral osteotomy correction for developmental coxavara: a new technique. J Pediatr Orthop 2005; 25(1):28-33. 